\title{
Surgical patients and the risk of malnutrition: preoperative screening requires assessment and optimization
}

\author{
Francesco Carli, MD, MPhil • Chelsia Gillis, RD, PhD
}

Received: 30 November 2020/ Accepted: 30 November 2020/Published online: 16 February 2021

(C) Canadian Anesthesiologists' Society 2021

\section{Malnutrition and surgical outcome}

The World Health Organization defines malnutrition as a deficiency, excess, or imbalance in a person's intake of energy and/or nutrients. ${ }^{1}$ The prevalence of malnutrition in hospitalized older adults in Canada is $40 \%^{2}$ and it is expected to increase as the population over the age of $75 \mathrm{yr}$ increases. ${ }^{3}$ Age, frailty, institutionalization, polypharmacy, general health decline (including physical function and cognition), depression, and dysphagia have been identified as risk factors for malnutrition. ${ }^{4}$ In surgical ${ }^{5}$ and nonsurgical populations, ${ }^{2}$ it is well recognized that malnutrition predisposes to high morbidity and mortality, and that malnutrition worsens when patients are admitted to Canadian hospitals. ${ }^{6}$

In the present issue, authors Gn et al. ${ }^{7}$ have conducted a retrospective cohort study of 1,033 older patients $\geq 65 \mathrm{yr}$ scheduled for elective surgery. Using the Malnutrition Universal Screening Tool (MUST) score, the authors reported that $11.9 \%$ of the patients studied were at risk of malnutrition (MUST $\geq 1$ ), and that $4.6 \%$ of these were at high risk (MUST $\geq 2$ ). After multivariable adjustment for sex, severity of surgery, comorbidities, and anemia,

F. Carli, MD, MPhil ( $\varangle)$

Department of Anesthesia, McGill University Health Centre, Montreal, QC, Canada

e-mail: franco.carli@mcgill.ca

Department of Anesthesia, McGill University Health Centre, Montreal General Hospital, 1650, Cedar Avenue, Room

D10.144, Montreal, QC H3G 1A4, Canada

C. Gillis, $\mathrm{RD}, \mathrm{PhD}$

Department of Anesthesia, McGill University Health Centre, Montreal, QC, Canada malnutrition was associated with significantly higher odds of postoperative complications and longer hospital stay. The results confirm what has been known for a long time i.e., malnutrition is negatively associated with postoperative outcome. The authors should be congratulated as they bring to the attention of anesthesiologists the importance of assessment of malnutrition in the elderly surgical population.

Nutritional problems in older adults often remain undetected or unaddressed, likely due to poor knowledge of malnutrition among medical staff. In recent decades, efforts have been made to incorporate teaching on nutrition into the undergraduate and postgraduate medical curricula to address this gap. ${ }^{8}$ Recognition and treatment of malnutrition remain the responsibility of every perioperative clinician when preparing and optimizing patients for surgery. Nutritional screening, assessment, and prompt treatment of deficits before surgery must be emphasized.

\section{Screening for malnutrition}

Perioperative physicians recognize malnutrition as an important issue, yet formal screening processes are observed in only $6-38 \%$ of surgical sites. ${ }^{9}-{ }^{11}$ Instead, concerned clinicians often use non-validated laboratory parameters and subjective assessments to "identify" malnourished patients. ${ }^{9,10}$ Informal screening procedures often fail to recognize malnutrition, ${ }^{12}$ possibly because clinicians erroneously pursue the phenotypic expression of malnutrition only. According to the Global Leadership Initiative on Malnutrition (GLIM), the diagnosis of malnutrition includes both the identification of a 
phenotype, expressed as loss of muscle mass or unintentional weight loss, and an etiology, including disease burden and anorexia. ${ }^{13,14}$ The phenotypic expression of malnutrition might not be recognizable through observation alone. Computed tomography studies of body composition have revealed that weight loss, muscle index and muscle attenuation (i.e., muscle quantity and quality) vary widely among all body mass index categories. ${ }^{15}$ It is for this reason that systematic screening for malnutrition drastically increases referrals to dietitians for further assessment and treatment. ${ }^{16}$

Screening tools, including the Canadian Nutrition Screening Tool $^{13}$ (validated in Canadian medical and surgical inpatients using the "gold standard" Subjective Global Assessment), and the Mini Nutritional AssessmentShort Form ${ }^{17}$ (validated against the Mini Nutritional Assessment used to diagnose malnutrition in older adults) can be used by trained non-nutrition personnel to identify patients at malnutrition risk. While the MUST, employed by Gn et al., ${ }^{7}$ is a validated screening tool endorsed by the European Society of Clinical Nutrition and Metabolism (ESPEN), the Mini Nutritional Assessment is recommended for use in older adults. ${ }^{18}$ Patients flagged as at risk for malnutrition can then be referred to dietitians who conduct comprehensive nutrition assessments for the purpose of diagnosing malnutrition and other nutritionrelated problems (Figure). Early screening using a validated tool, such as at the first surgical visit, offers a window of opportunity to intervene with a targeted or specialized nutrition intervention that could improve patient outcomes and experiences.

\section{Action is needed: nutritional intervention beyond screening and assessment}

In the current trajectory of perioperative care, the preoperative clinic remains a logical place for further nutritional assessment of patients identified to be at risk of malnutrition and to plan appropriate intervention. At this stage, patients diagnosed as at risk of malnutrition require a nutritional assessment by a dietitian who will determine the degree of nutrition risk, complete a comprehensive nutritional examination, and propose a course of action aiming at a structured, personalized nutritional intervention. With a high prevalence of malnutrition in older populations scheduled for surgery, it makes sense to emphasize and to value the role of the dietitian in the preoperative clinic. A concept of nutritional prehabilitation is emerging in perioperative medicine in view of the potential impact of high-quality nutrition on postoperative outcome. ${ }^{19}$ Malnourished patients are most likely to benefit from nutrition therapy, particularly older patients who develop insulin resistance and are unable to enhance tissue protein synthesis in response to protein intake. Cancer or inflammation further impairs nutrient utilization and is associated with nutrition-impact symptoms that impede adequate food intake, and thus may require targeted intervention. A multi-disciplinary team led by the anesthesiologist as the perioperative clinician, together with the surgeon, dietitian, exercise physiologist, and psychologist, can identify prognostic determinants of malnutrition and plan a series of interventions to restore and maintain nutritional status, muscle mass, and strength. As a result, surgery may have to be postponed to enhance anabolism and attenuate further catabolism, particularly in high risk patients.

Preoperative optimization of nutritional status using a risk-stratified approach based on nutritional screening and assessment can target patients that will benefit most and use resources wisely. ${ }^{20}$ Although seven to 14 days of nutrition optimization has been observed to improve postoperative outcomes, such as length of hospital stay, ${ }^{19,21}$ earlier screening and assessment affords greater possibility for nutrition care management and success. Nutritional treatment, particularly as a component of multimodal prehabilitation, represents an opportunity to mitigate poor surgical outcomes and the acute catabolic effects of surgery.

\section{Patients chirurgicaux et risque de malnutrition : le dépistage préopératoire nécessite évaluation et optimisation subséquentes}

\section{Malnutrition et devenirs chirurgicaux}

L'Organisation mondiale de la Santé définit la malnutrition comme une carence, un excès ou un déséquilibre dans la consommation d'énergie et/ou de nutriments d'une personne. ${ }^{1}$ La prévalence de la malnutrition chez les personnes âgées hospitalisées au Canada est de $40 \%{ }^{2}$ et on s'attend à ce qu'elle augmente au fur et à mesure que la population âgée de plus de 75 ans augmente. ${ }^{3}$ L'âge, la fragilité, l'institutionnalisation, la polypharmacie, le déclin général de la santé (y compris la capacité physique fonctionnelle et la cognition), la perte d'intérêt pour la vie et la dysphagie ont été identifiés comme des facteurs de risque de malnutrition. ${ }^{4} \mathrm{Chez}$ les populations chirurgicales ${ }^{5}$ et non chirurgicales, ${ }^{2}$ il a été établi que la malnutrition prédisposait à une morbidité et à une mortalité élevées et 
que, chez les patients admis dans les hôpitaux canadiens, elle détériorait encore davantage l'état nutritionnel. ${ }^{6}$

Dans le présent numéro, les auteurs Gn et coll. ${ }^{7}$ ont mené une étude de cohorte rétrospective chez 1033 patients âgés $\geq 65$ ans devant subir une chirurgie non urgente. À l'aide du score de l'Outil de dépistage universel de la malnutrition (MUST), les auteurs rapportent que 11,9 $\%$ des patients à l'étude étaient à risque de malnutrition (MUST $\geq 1$ ) et que 4,6\% d'entre eux étaient à haut risque (MUST $\geq 2$ ). Après ajustement multivariable pour tenir compte du sexe, de l'importance de la chirurgie, des comorbidités et de l'anémie, la malnutrition a été associée à un risque significativement plus élevé de complications postopératoires et d'hospitalisation prolongée. Ces résultats confirment ce que nous savons depuis longtemps - c'est-àdire, que la malnutrition est négativement associée au devenir postopératoire. Il convient de féliciter les auteurs car ils attirent l'attention des anesthésiologistes sur l'importance de l'évaluation de la malnutrition chez la population chirurgicale âgée.

Chez les personnes âgées, les problèmes de nutrition restent souvent non dépistés ou non abordés, probablement en raison d'une piètre connaissance de la malnutrition par le personnel médical. Afin de combler ces lacunes, des efforts ont été déployés au cours des dernières décennies pour intégrer l'enseignement de la nutrition dans les programmes médicaux de premier et de second cycle. ${ }^{8}$
La reconnaissance et le traitement de la malnutrition demeurent la responsabilité de chaque clinicien périopératoire lors de la préparation et de l'optimisation des patients pour la chirurgie. Il faut mettre l'emphase sur le dépistage, l'évaluation, et le traitement prompt des déficits nutritionnels avant une chirurgie.

\section{Dépister la malnutrition}

On rapporte que les médecins périopératoires considèrent systématiquement la malnutrition comme un problème important; pourtant, seuls 6 à $38 \%$ des sites chirurgicaux disposent de processus formels de dépistage. ${ }^{9-11}$ Au lieu de cela, les cliniciens inquiets ont souvent recours à des paramètres de laboratoire non validés et des évaluations subjectives pour «identifier» les patients souffrant de malnutrition. ${ }^{9,10}$ Les processus de dépistage informels ne reconnaissent souvent pas bien la malnutrition, ${ }^{12}$ peut-être parce que les cliniciens n'évaluent, à tort, que l'expression phénotypique de la malnutrition. Le diagnostic de malnutrition comprend à la fois l'identification d'un phénotype, exprimé en tant qu'une perte de masse musculaire ou de poids involontaire, et une étiologie, qui inclut le fardeau de la maladie et l'anorexie. ${ }^{13,14}$ Fait important, l'expression phénotypique de la malnutrition pourrait ne pas être identifiable par l'observation

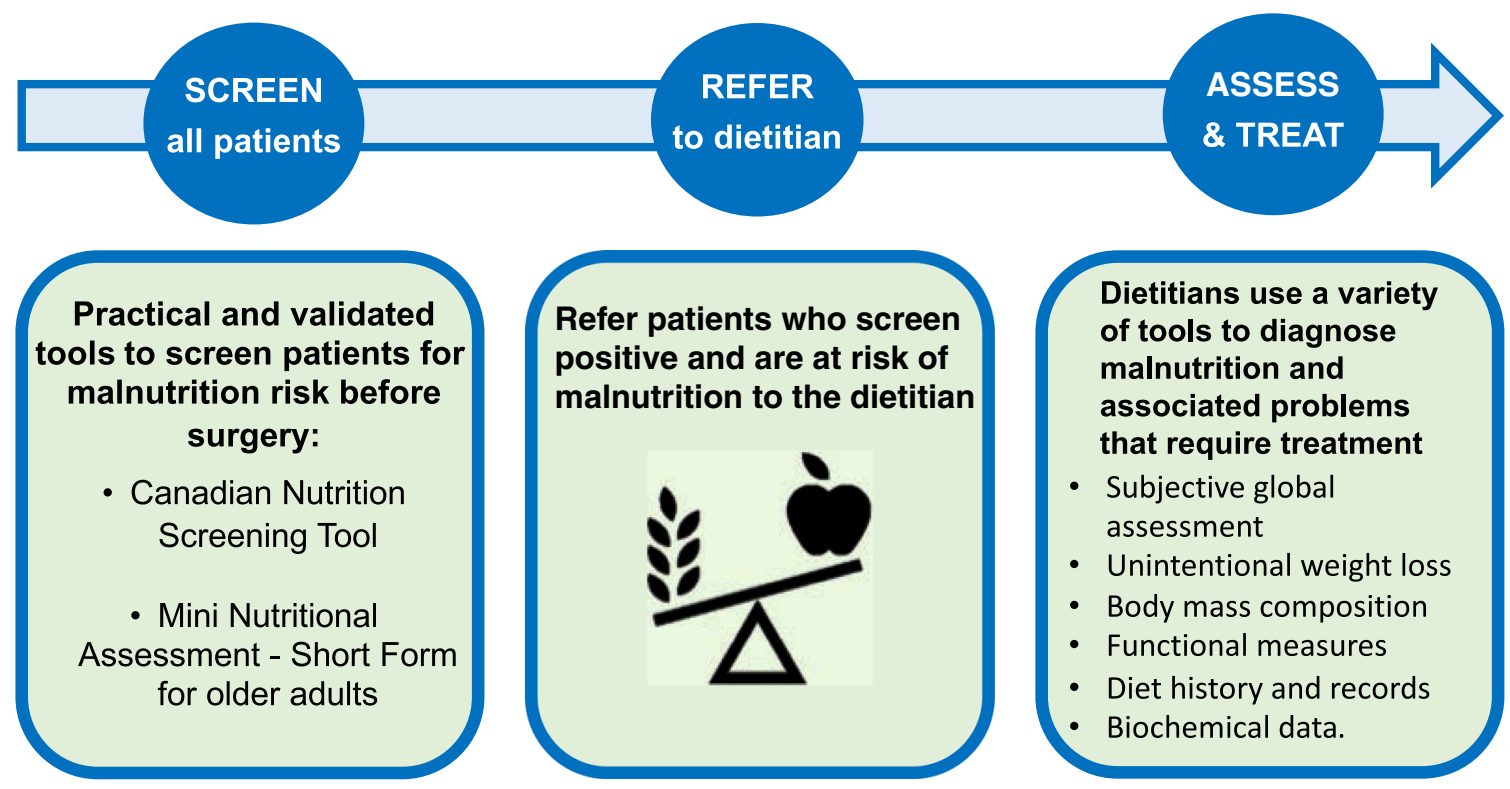

Figure All patients should be screened early for malnutrition risk before surgery. A positive screen necessitates a referral to a dietitian for an assessment of nutritional status. A dietitian can diagnose malnutrition and other nutrition problems that require treatment before surgery.Tous les patients devraient être dépistés rapidement pour évaluer leur risque de malnutrition avant chirurgie. Un dépistage positif nécessite une référence à un diététiste afin de réaliser une évaluation de l'état nutritionnel. Un diététiste peut diagnostiquer la malnutrition et d'autres problèmes nutritionnels qui nécessitent un traitement avant la chirurgie. 
seulement. Les études de tomodensitométrie de la composition corporelle ont indiqué que la perte de poids, l'indice musculaire et l'atténuation musculaire varient considérablement et ce, dans toutes les catégories d'indice de masse corporelle. ${ }^{15}$ C'est pour cette raison que le dépistage systématique de la malnutrition augmente considérablement les références aux diététistes afin qu'ils procèdent à une évaluation et un traitement plus poussés. ${ }^{16}$

Les outils de dépistage, y compris l'Outil canadien de dépistage nutritionnel ${ }^{13}$ (validé chez les patients hospitalisés médicaux et chirurgicaux canadiens à l'aide de l'Évaluation globale subjective comme référence (gold standard)) et le Formulaire abrégé de la Mini évaluation nutritionnelle (Mini Nutritional Assessment-Short Form) ${ }^{17}$ (validé à l'aide du questionnaire Mini Nutritional Assessment (MNA) utilisé pour diagnostiquer la malnutrition chez les personnes âgées) peuvent être utilisés par du personnel sans formation en nutrition pour identifier les patients courant un risque de malnutrition. Bien que le MUST, employé par Gn et coll., ${ }^{7}$ soit un outil de dépistage validé et approuvé par La Société Européenne de Nutrition Clinique et Métabolisme (ESPEN), le MNA est l'outil recommandé pour les personnes âgées. ${ }^{18}$ Les patients identifiés comme courant un risque de malnutrition peuvent ensuite être dirigés vers des diététistes qui effectuent des évaluations nutritionnelles complètes dans le but de diagnostiquer la malnutrition et d'autres problèmes liés à la nutrition (figure). Un dépistage précoce, réalisé à l'aide d'un outil validé, lors de la première visite chirurgicale par exemple, offre une occasion d'agir via une intervention nutritionnelle ciblée ou spécialisée qui pourrait améliorer les devenirs et les expériences des patients.

\section{Des mesures s'imposent : l'intervention nutritionnelle au-delà du dépistage et de l'évaluation}

Dans la trajectoire actuelle des soins périopératoires, la clinique préopératoire demeure un choix logique pour réaliser une évaluation nutritionnelle plus poussée des patients identifiés comme étant à risque de malnutrition et pour planifier une intervention appropriée. À ce stade, les patients diagnostiqués comme courant un risque de malnutrition ont besoin d'une évaluation nutritionnelle par un diététiste qui déterminera le niveau de risque nutritionnel, effectuera un examen nutritionnel complet et proposera une ligne de conduite visant une intervention nutritionnelle à la fois structurée et personnalisée. Étant donné la prévalence élevée de malnutrition chez les populations âgées devant subir une chirurgie, il est logique de souligner et de valoriser le rôle du diététiste dans la clinique préopératoire. En médecine périopératoire, le concept de préhabilitation nutritionnelle émerge en raison de l'impact potentiel d'une nutrition de haute qualité sur les devenirs postopératoires. ${ }^{19}$ Les patients souffrant de malnutrition sont les plus susceptibles de bénéficier d'une thérapie nutritionnelle, en particulier les patients âgés qui développent une résistance à l'insuline et sont incapables d'augmenter la synthèse des protéines tissulaires en réponse à un apport en protéines. Le cancer ou l'inflammation entrave encore plus l'utilisation des nutriments et pourrait nécessiter une intervention ciblée. Une équipe multidisciplinaire dirigée par l'anesthésiologiste en tant que clinicien périopératoire, de concert avec le chirurgien, le diététiste, le physiologiste de l'exercice et le psychologue, peut identifier les déterminants pronostiques de la malnutrition et planifier une série d'interventions afin de rétablir et maintenir l'état nutritionnel, la masse musculaire et la force. En conséquence, il est possible que la chirurgie doive être reportée pour augmenter l'anabolisme et atténuer davantage le catabolisme, particulièrement chez les patients à haut risque.

L'optimisation préopératoire de l'état nutritionnel à l'aide d'une approche de stratification du risque basée sur le dépistage et l'évaluation nutritionnels peut cibler les patients qui bénéficieront le plus d'une telle approche et utiliseront les ressources à bon escient. ${ }^{20}$ Bien qu'il ait été observé que sept à 14 jours d'optimisation nutritionnelle soient nécessaires pour améliorer les devenirs postopératoires tels que la durée d'hospitalisation, ${ }^{19,21}$ un dépistage et une évaluation plus précoces offrent une plus grande possibilité de gestion et de succès des soins nutritionnels. Le traitement nutritionnel, en particulier dans le cadre d'une préhabilitation multimodale, représente une occasion de réduire les mauvais devenirs chirurgicaux et les effets cataboliques aigus de la chirurgie.

Disclosures None.

Funding statement None.

Editorial responsibility This submission was handled by Dr. Alana M. Flexman, Associate Editor, Canadian Journal of Anesthesia.

Déclaration Aucune.

Déclaration de financement Aucune.

Responsabilité éditoriale Cet article a été traité par Dr Alana M Flexman, rédactrice adjointe, Journal canadien d'anesthésie.

\section{References}

1. World Health Organization. Integrated Care for Older People (ICOPE). Guidelines on community-level interventions to manage declines in intrinsic capacity - 2017. Available from URL: https://apps.who.int/iris/bitstream/handle/10665/258981/ 
9789241550109-eng.pdf;jsessionid=493ACCB96B8EC7D4900A FF17B32CB1CE? sequence=1 (accessed 30 November 2020).

2. Curtis LJ, Bernier P, Jeejeebhoy K, et al. Costs of hospital malnutrition. Clin Nutr 2017; 36: 1391-6.

3. Muscedere J, Andrew MK, Bagshaw SM, et al. Screening for frailty in Canada's health care system: a time for action. Can J Aging 2016; 35: 281-97.

4. Fávaro-Moreira NC, Krausch-Hofmann S, Matthys $C$, et al. Risk factors for malnutrition in older adults: a systematic review of the literature based on longitudinal data. Adv Nutr 2016; 7: 507-22.

5. Sun $Z$, Kong $X J$, Jing $X$, Deng $R J$, Tian $Z B$. Nutritional risk screening 2002 as a predictor of postoperative outcomes in patients undergoing abdominal surgery: a systematic review and meta-analysis of prospective cohort studies. PloS One 2015; DOI: https://doi.org/10.1371/journal.pone.0132857.

6. Keller H, Allard J, Vesnaver E, et al. Barriers to food intake in acute care hospitals: a report of the Canadian Malnutrition Task Force. J Hum Nutr Diet 2015; 28: 546-57.

7. Gn YM, Abdullah HR, Loke W, Sim YE. Prevalence and risk factors of preoperative malnutrition risk in older patients and its impact on surgical outcomes: a retrospective observational. Can J Anesth 2021; DOI: https://doi.org/10.1007/s12630-021-01933-3.

8. Crowley J, Ball L, Hiddink GJ. Nutrition in medical education: a systematic review. Lancet Planet Health 2019; DOI: https://doi. org/10.1016/S2542-5196(19)30171-8.

9. Williams JD, Wischmeyer PE. Assessment of perioperative nutrition practices and attitudes - a national survey of colorectal and GI surgical oncology programs. Am J Surg 2017; 213: 1010-8.

10. Grass $F$, Cerantola $Y$, Schäfer $M$, Müller S, Demartines $N$, Hübner $M$. Perioperative nutrition is still a surgical orphan: results of a Swiss-Austrian survey. Eur J Clin Nutr 2011; 65: 642-7.

11. Breuer JP, Langelotz C, Paquet P, et al. Perioperative nutrition a nationwide web-based survey of German surgery departments (German). Zentralbl Chir 2013; 138: 622-9.

12. Aktas A, Walsh D, Galang $M$, et al. Underrecognition of malnutrition in advanced cancer: the role of the dietitian and clinical practice variations. Am J Hosp Palliat Care 2017; 34: 547-55.

13. Laporte $M$, Keller $H$, Payette $H$, et al. Validity and reliability of the new Canadian Nutrition Screening Tool in the 'real-world' hospital setting. Eur J Clin Nutr 2015; 69: 558-64.

14. Cederholm T, Jensen GL, Correia MI, et al. GLIM criteria for the diagnosis of malnutrition - a consensus report from the global clinical nutrition community. Clin Nutr 2019; 38: 1-9.

15. Martin L, Birdsell L, Macdonald N, et al. Cancer cachexia in the age of obesity: skeletal muscle depletion is a powerful prognostic factor, independent of body mass index. J Clin Oncol 2013; 31 : 1539-47.

16. Frew E, Cant R, Sequeira J. Capturing the data: nutrition risk screening of adults in hospital. Nutrients 2010; 2: 438-48.

17. Kaiser MJ, Bauer JM, Ramsch C, et al. Validation of the Mini Nutritional Assessment short-form (MNA-SF): a practical tool for identification of nutritional status. J Nutr Health Aging 2009; 13: 782-8.

18. Cederholm T, Barazzoni R, Austin P, et al. ESPEN guidelines on definitions and terminology of clinical nutrition. Clin Nutr 2017; 36: 49-64.

19. Gillis C, Buhler $K$, Bresee $L$, et al. Effects of nutritional prehabilitation, with and without exercise, on outcomes of patients who undergo colorectal surgery: a systematic review and meta-analysis. Gastroenterology 2018; 155: 391-410.e4.

20. Macmillan Cancer Support. Principles and guidance for prehabilitation within the management and support of people with cancer. Available from URL: https://www.macmillan.org. uk/_images/prehabilitation-for-people-with-cancer_tcm9-353994. pdf (accessed November 2020).

21. Weimann A, Braga M, Carli $F$, et al. ESPEN guideline: Clinical nutrition in surgery. Clin Nutr 2017; 36: 623-50.

Publisher's Note Springer Nature remains neutral with regard to jurisdictional claims in published maps and institutional affiliations. 